\title{
Hipertrofia ventricular izquierda y valvulopatías en la enfermedad renal crónica. Prevalencia y factores de riesgo asociados
}

\author{
Xosé González-Cancela1, Gabriel Parma², Patricia Larre-Borges", Lucía Florio² y Liliana Gadola ${ }^{1 *}$ \\ ${ }^{1}$ Centro de Nefrología; ${ }^{2}$ Centro Cardiovascular. Hospital de Clínicas, Universidad de la República, Montevideo, Uruguay
}

\begin{abstract}
Resumen
La enfermedad cardiovascular es la principal causa de muerte en la enfermedad renal crónica (ERC). Objetivos: Analizar la prevalencia y los factores de riesgo de hipertrofia ventricular izquierda (HVI) y valvulopatías en la ERC estadios I-V (sin diálisis). Métodos: Análisis retrospectivo de datos clínicos, bioquímicos y ecocardiográficos de pacientes con ERC del 1 de enero de 2010 al 30 de junio de 2016. Aprobado por Comité de Ética. Resultados: Se incluyeron 350 pacientes, $52.3 \%$ mujeres, edad mediana 65 años. Se analizaron dos grupos, ERC estadio I-II (30.3\%) vs. estadio III-V (69.7\%). Se observó una elevada frecuencia de remodelación cardíaca, mayor en estadio III-V. El menor filtrado glomerular estimado (FGe) se asoció en análisis multivariado (Cox) a mayor masa ventricular izquierda (OR: 1.002; IC 95\%: 1.000-1.003; $p=0.048$ ), independiente de la edad, la hipertensión y la diabetes. En el subgrupo con HVI excéntrica, el FGe y la fracción de eyección del ventrículo izquierdo eran menores, y en el subgrupo con HVI concéntrica, la presión arterial diastólica era más elevada. Las valvulopatías fueron más frecuentes en estadio III-V, principalmente insuficiencia mitral (38.5\% vs. 24.5\%) y aórtica (28.6\% vs. 12.3\%). Conclusiones: Las anomalías morfológicas cardíacas fueron más frecuentes en estadios de ERC III-V, y el descenso del FGe fue un factor de riesgo independiente de HVI.
\end{abstract}

Palabras clave: Enfermedad renal crónica. Hipertrofia ventricular izquierda. Índice de masa ventricular izquierda. Valvulopatías.

\section{Left ventricular hypertrophy and heart valve disease in chronic kidney disease. Prevalence and risk factors}

\section{Abstract}

Cardiovascular disease is the main cause of death among chronic kidney disease (CKD) patients. Aims: To analyze heart disease prevalence and risk factors in a CKD cohort). Methods: A retrospective study of CKD patients' clinical, lab and echocardiography data were done, between January 1, 2010 and June 30, 2016. All CKD patients with a transthoracic echocardiography data were included. The study was approved by the Ethical Committee. Results: Three hundred fifty patients were included, $52.3 \%$ females, median age 65 years. Two groups were analyzed: CKD stage I-II (30.3\%) vs stage III-V (69.7\%). A higher frequency of cardiac remodeling were observed in stage III-V. The lower estimated Glomerular filtration rate (eGFR) the higher left ventricular (LV) mass observed (Odd ratio: 1.002; Cl 95\%: 1.000-1.003; $p=0.048$ ) independently of age, hy-

\section{Correspondencia:}

*Liliana Gadola

E-mail: lilianagad@gmail.com
Fecha de recepción: 03-07-2019

Fecha de aceptación: 06-02-2020

DOI: 10.24875/NEFRO.20000077
Disponible en internet: 03-04-2020

Nefro Latinoam. 2020;17:34-42 www.nefrologialatinoamericana.com

2444-9032/@ 2020 Sociedad Latinoamericana de Nefrología e Hipertensión. Publicado por Permanyer. Este es un artículo open access bajo la licencia CC BY-NC-ND (http://creativecommons.org/licenses/by-nc-nd/4.0/). 
pertension and diabetes. The subgroup with LV mass index data and Eccentric LV hypertrophy (LVH) was associated with lower eGFR and LV ejection fraction and concentric LVH was associated with higher diastolic blood pressure. Heart valve disease were more frequent in CKD stage III-V, mainly mitral (38.5\% vs. $24.5 \%)$ and aortic (28.6\% vs. $12.3 \%$ ) regurgitation. Conclusions: Cardiac disease (auricular, ventricular and valvular) were more frequent among CKD III-V patients and a lower eGFR was independently associated with LVH.

Key words: Chronic kidney disease. Left ventricular hypertrophy. Left ventricular mass index. Heart valve disease.

\section{Introducción}

La enfermedad renal crónica (ERC) tiene una elevada incidencia y prevalencia ${ }^{1-3}$, con una elevada morbimortalidad cardiovascular. La enfermedad cardiovascular (ECV) aumenta a medida que disminuye el filtrado glomerular-1-8, y es la principal causa de muerte en esta población?

Se han descrito factores de riesgo de ECV tradicionales y no tradicionales, vinculados a toxinas urémicas, injuria nitrooxidativa, inflamación ${ }^{4-7}$. En Uruguay, los datos del Registro del Programa de Salud Renal ${ }^{2}$ reportan que el $40 \%$ de los pacientes presentan antecedentes cardiovasculares al ingreso al programa, y que la prevalencia de cardiopatía aumenta con cada etapa de la ERC ${ }^{8}$.

La hipertrofia ventricular izquierda $(\mathrm{HVI})$ es altamente prevalente en portadores de ERC $(50-80 \%$, según el estadio) $^{9-11}$, por estímulos hemodinámicos (hipertensión arterial [HTA], sobrehidratación), inflamación e injuria oxidativa ${ }^{12}$, anemia, exceso de mineralocorticoides $^{13}$, alteraciones del metabolismo óseo-mineral ${ }^{14,15}$. Existen menos reportes sobre la frecuencia de afectación valvular (calcificada o no) en el curso de la ERC $y$ de sus factores de riesgo ${ }^{16,17}$.

\section{Objetivos}

Analizar la prevalencia y factores de riesgo de HVI y de valvulopatías en una población con enfermedad renal crónica en estadios I a V (sin diálisis).

\section{Métodos}

Estudio retrospectivo de la cohorte del Centro de Nefrología del Hospital de Clínicas incluidos en el Programa de Salud Renal del Uruguay (PSRU) ${ }^{2}$, en el periodo del 1 de enero de 2010 al 30 de junio de 2016, con un ecocardiograma Doppler (ETT) de control realizado en este periodo. Los criterios de inclusión al Registro del PSRU son adultos con alteraciones renales persistentes por más de 3 meses: filtrado glomerular estimado (FGe) menor de $60 \mathrm{ml} / \mathrm{min} / 1.73 \mathrm{~m}^{2}$, o proteinuria mayor de $300 \mathrm{mg} /$ día o albuminuria mayor de $30 \mathrm{mg} / \mathrm{día}$ en diabéticos, asistidos por nefrólogos. Los ETT fueron realizados por dos cardiólogos experimentados pertenecientes al Centro Cardiovascular del Hospital de Clínicas. Utilizaron un ecógrafo Siemens, Aqusson Secuoia C512, con transductor ecocardiográfico de $3.5 \mathrm{mHz}$.

Se registraron datos clínicos (sexo, edad, peso, talla, nefropatía y comorbilidades), bioquímicos (creatininemia, proteinuria, hemoglobinemia, fosforemia, calcemia, potasemia, colesterolemia) y ecocardiográficos (fecha de realización del ecocardiograma, fracción de eyección del ventrículo izquierdo [FEVI]), patrón de llenado, diámetro diastólico del VI, presencia de hipertrofia ventricular izquierda, espesor del septum y de la pared posterior, cálculo de masa del ventrículo izquierdo, índice de masa ventricular [IMV], espesor relativo de la pared ventricular izquierda [RWT], área de la aurícula izquierda [AI], diámetro de la Al, calcificación valvular, presencia y tipo de valvulopatía). Los datos fueron concomitantes a cada ecocardiograma.

Se definió ERC según K/DIGO ${ }^{18}$; HTA según, el VII informe del Joint National Committee ${ }^{19}$; presión arterial igual o superior a 140/90 mmHg; filtrado glomerular estimado calculado por la fórmula CDK-EPI ${ }^{6}$; HVI según los parámetros ecocardiográficos con base en el aumento del espesor parietal $(>11 \mathrm{~mm})$ o de la masa ventricular según la fórmula estandarizada por la American Society of Echocardiography ${ }^{11}$, HVI si la masa era mayor a $150 \mathrm{~g}$ en la mujer y $200 \mathrm{~g}$ en el hombre, 0 estandarizado al índice de masa ventricular (con base en el peso y la talla) que define la HVI si es mayor de $88 \mathrm{~g} / \mathrm{m}^{2}$ en la mujer y $102 \mathrm{~g} / \mathrm{m}^{2}$ en el hombre. El estudio de la geometría ventricular se completa mediante el cálculo del RWT según la fórmula: $(2 \mathrm{x}$ grosor de la pared posterior)/(diámetro interno $\mathrm{VI}$ al final de la diástole $)^{11}$. Permite la categorización del aumento de la masa del $\mathrm{VI}$, ya sea como hipertrofia concéntrica (RWT > 0.42) o excéntrica (RWT $\leq 0.42$ ), o la identificación del remodelado concéntrico (masa normal con RWT aumentado). 


\section{Aspectos éticos}

Este estudio fue aprobado por el Comité de Ética del Hospital de Clínicas, Facultad de Medicina (Universidad de la República).

Los pacientes incluidos en el Registro del PSRU firmaron consentimiento informado para autorizar el análisis de sus datos no identificados. Se cumplieron las normas internacionales, y nacionales según la Ley de Protección de Datos Personales y Acción de Habeas Data, N. ${ }^{\circ} 18,331$.

\section{Análisis estadístico}

Para las variables cuantitativas se calculó mediana y rango intercuartílico (Pc 25-75 = Q1-Q3), para las variables categóricas se calcularon porcentajes. La comparación entre grupos se realizó mediante tests no paramétricos (Mann Whitney y Kruskall-Wallis) para las variables cuantitativas y test de $\mathrm{Chi}^{2}$ para las variables categóricas. Se consideró significativo un valor $p<0.05$. Se realizó un análisis uni y multivariado mediante regresión logística; se incluyeron en el modelo multivariado aquellas variables que presentaban un valor $p<0.05$ en el univariado. Se utilizó el software IBM $^{\circledR}$ SPSS Statistics ${ }^{\circledR}$, versión 22.

\section{Resultados}

Se analizaron 350 pacientes, 183 mujeres (52.3\%), con una edad mediana de 65 años (Q1-Q3: 54-75), (entre 18 y 92 años). Las nefropatías más frecuentes fueron: nefropatía vascular (18.3\%), túbulo intersticiales $(9.6 \%)$, glomerulopatías/enfermedades autoinmunes $(7.9 \%)$ y nefropatía diabética $(7 \%)$. Las comorbilidades registradas fueron: hipertensión arterial $(58.6 \%)$, diabetes $(28.6 \%)$, dislipemia $(4.9 \%)$ y tabaquismo actual (3.5\%). El $10.9 \%$ presentaban cardiopatía isquémica, el 7.1\%, insuficiencia cardíaca, el 3\%, arteriopatía obstructiva crónica de los miembros inferiores, el $3.3 \%$, evento cerebrovascular (Tabla 1).

Se dividió a la población estudiada en dos grupos según el filtrado glomerular estimado simultáneo al ecocardiograma: grupo con $\mathrm{FGe} \geq 60 \mathrm{ml} / \mathrm{min} / 1.73 \mathrm{~m}^{2}$ (estadio ERC I-II) o con FGe $<60 \mathrm{ml} / \mathrm{min} / 1.73 \mathrm{~m}^{2}$ (ERC III-V sin diálisis).

La mediana del FGe de la población global fue de $48.2 \mathrm{ml} / \mathrm{min} / 1.73 \mathrm{~m}^{2}$ (33.2-63.6) y los estadios de ERC eran: $8.9 \%$ en estadio I, $21.4 \%$ en el II, $51.4 \%$ en el III, $15.1 \%$ en el IV y $3.1 \%$ en el V (Tabla 1 ).
Tabla 1. Características de la población estudiada

\begin{tabular}{|c|c|}
\hline Población estudiada (n) & 350 \\
\hline Sexo (femenino/masculino) $(\%)$ & $52.3 / 47.7$ \\
\hline Edad (años) mediana (Pc 25-75) & $65(54-75)$ \\
\hline $\mathrm{FGe}\left(\mathrm{ml} / \mathrm{min} / 1.73 \mathrm{~m}^{2}\right)$ mediana (Pc 25-75) & $48.2(33.2-63.6)$ \\
\hline Nefropatía & $(\%)$ \\
\hline Vascular & 18.3 \\
\hline Diabética & 7 \\
\hline Glomerulopatía/enfermedades autoinmunes & 7.9 \\
\hline Nefropatía tubulointersticial & 9.6 \\
\hline Otras & 40.3 \\
\hline Sin dato & 16.9 \\
\hline Etapa ERC (en el primer ecocardiograma) & $(\%)$ \\
\hline I & 8.9 \\
\hline II & 21.4 \\
\hline III & 51.4 \\
\hline IV & 15.1 \\
\hline V & 3.1 \\
\hline $\begin{array}{l}\text { Antecedentes de factores de riesgo } \\
\text { cardiovascular }\end{array}$ & $(\%)$ \\
\hline Hipertensión arterial & 58.6 \\
\hline Diabetes & 28.6 \\
\hline Dislipemia & 4.9 \\
\hline Tabaquismo & 3.5 \\
\hline Antecedentes de enfermedad cardiovascular & $(\%)$ \\
\hline Cardiopatía isquémica & 10.9 \\
\hline Insuficiencia cardíaca & 7.1 \\
\hline $\begin{array}{l}\text { Arteriopatía periférica de miembros } \\
\text { inferiores }\end{array}$ & 3 \\
\hline Evento cerebrovascular & 3.3 \\
\hline
\end{tabular}

ERC: enfermedad renal crónica; FGe: filtrado glomerular estimado por CKD-EPI.

Se realizaron 350 ETT (Tabla 2). La mediana (Pc 25-75) de la FEVI fue del 60\% (55-65), destacando que el $10 \%$ de los pacientes presentaron una FEVI menor del $40 \%$. La mediana del septum interventricular fue de $11 \mathrm{~mm}$ (10-12) y la de la pared posterior de $10 \mathrm{~mm}$ (9-11), significativamente mayores en el grupo con ERC III-V. La frecuencia de HVI varió según la definición: $42 \%$ según el espesor parietal, $46 \%$ evaluada por masa ventricular y $60.6 \%$ evaluada por IMV, 
Tabla 2. Parámetros simultáneos clínicos, bioquímicos y del ecocardiograma $(n=350)$

\begin{tabular}{|c|c|c|c|c|}
\hline & Población global & Grupo ERC I-II & Grupo ERC III-V & Test $/ p$ \\
\hline $\mathrm{N}(\%)$ & $350(100 \%)$ & $106(30.3 \%)$ & $244(69.7 \%)$ & \\
\hline Edad (años) mediana (Pc 25-75) & $65.0(54-75)$ & $57.5(19.0)$ & $69.0(17.0)$ & ${ }^{*} p=0.000$ \\
\hline Sexo (femenino/masculino) & $183 / 167$ & $59 / 47$ & $124 / 120$ & ${ }^{\dagger} \mathrm{NS}$ \\
\hline Diabetes (\%) & $100(28.6 \%)$ & $30(28.3 \%)$ & $70(28.6 \%)$ & ${ }^{*} \mathrm{NS}$ \\
\hline PAS (mmHg) mediana (Pc 25-75) & $130(120-149)$ & $127(120-146)$ & $130(120-150)$ & ${ }^{*} \mathrm{NS}$ \\
\hline PAD (mmHg) mediana (Pc 25-75) & $76(66-83)$ & $77(69-82)$ & $74.5(65-83)$ & ${ }^{*} \mathrm{NS}$ \\
\hline Proteinuria (g/l) mediana (Pc 25-75) & $0.01(0.00-0.33)$ & $0.1(0.0-0.3)$ & $0.1(0.0-0.37)$ & ${ }^{*} \mathrm{NS}$ \\
\hline Potasemia (mEq/l) mediana (Pc 25-75) & $4.8(4.4-5.1)$ & $4.4(4.1-5.0)$ & $4.9(4.6-5.3)$ & ${ }^{*} p=0.004$ \\
\hline Hemoglobinemia (g/dl) mediana (Pc 25-75) & $13.1(12.2-14.2)$ & $13.7(13-14.3)$ & $12.9(11.9-13.7)$ & ${ }^{*} p=0.000$ \\
\hline Fosforemia (mg/dl) mediana (Pc 25-75) $(\mathrm{n}=135)$ & $3.6(3.2-4.0)$ & $3.5(3.3-3.8)$ & $3.7(3.2-4.0)$ & ${ }^{*} \mathrm{NS}$ \\
\hline Calcemia (mg/dl) mediana (Pc 25-75) $(\mathrm{n}=146)$ & $9.5(9.0-9.6)$ & $9.4(8.8-9.7)$ & $9.5(8.9-9.5)$ & ${ }^{*} \mathrm{NS}$ \\
\hline Colesterolemia (mg/dl) (Pc 25-75) $(\mathrm{n}=77)$ & $171(154-200)$ & $160(138-212)$ & $186(156-210)$ & ${ }^{*} \mathrm{NS}$ \\
\hline Trigliceridemia $(\mathrm{mg} / \mathrm{dl})(\mathrm{Pc} 25-75)(\mathrm{n}=77)$ & $159(139-202)$ & $130(76-161)$ & $190(140-233)$ & ${ }^{*} \mathrm{NS}$ \\
\hline HDL-col (mg/dl) (Pc 25-75) (n=77) & $44(35-53)$ & $50(42-59)$ & $39(32-49)$ & ${ }^{*} \mathrm{NS}$ \\
\hline LDL-col (mg/dl) (Pc 25-75) (n= 77) & $102(87-118)$ & $93(70-109)$ & $109(88-124)$ & ${ }^{*} \mathrm{NS}$ \\
\hline \multicolumn{5}{|l|}{ Ecocardiograma Doppler } \\
\hline FEVI (\%) mediana (Pc 25-75) & $60(55-65)$ & $60(60-65)$ & $60(55-65)$ & ${ }^{*} p=0.001$ \\
\hline $\mathrm{FEVI}<40 \%(\mathrm{n}-\%)$ & $35(10 \%)$ & $6(5.7 \%)$ & $29(11.9 \%)$ & ${ }^{\dagger} \mathrm{NS}$ \\
\hline DDVI (mm) mediana (Pc 25-75) & $48(43-52)$ & $45(42-49)$ & $49(43-53)$ & ${ }^{*} p=0.001$ \\
\hline PP (mm) mediana (Pc 25-75) & $10(9-11)$ & $10(9-11)$ & $10(10-12)$ & ${ }^{*} p=0.001$ \\
\hline SVI (mm) mediana (Pc 25-75) & $11(10-12)$ & $10(9-12)$ & $11(10-12)$ & ${ }^{*} p=0.005$ \\
\hline HVI por espesor parietal (\%) & $147(42.0 \%)$ & $35(33.0 \%)$ & $112(45.9 \%)$ & ${ }^{\dagger} p=0.033$ \\
\hline Masa VI (g) mediana (Pc 25-75) & $181(142-232)$ & $157(120-200)$ & $195(156-234)$ & ${ }^{*} p=0.000$ \\
\hline Al área (mm) (Pc 25-75) & $20(17-24)$ & $18(15-22)$ & $21(18-24)$ & ${ }^{*} p=0.001$ \\
\hline Al diámetro (mm) (Pc 25-75) & $37(32-41)$ & $35(31-39)$ & $38(34-42)$ & ${ }^{*} p=0.000$ \\
\hline IMV $\left(\mathrm{g} / \mathrm{mt}^{2}\right)(\mathrm{n}=160)$ mediana $(\operatorname{Pc} 25-75)$ & $102(86.5-127.5)$ & $92(76-115)$ & $107.5(91.5-130)$ & ${ }^{*} p=0.004$ \\
\hline RWT $(n=160)$ mediana $(P c 25-75)$ & $0.42(0.37-0.48)$ & $0.42(0.38-0.46)$ & $0.42(0.37-0.48)$ & ${ }^{*} \mathrm{NS}$ \\
\hline HVI por masa VI (n-\%) & $161(46 \%)$ & $36(33.3 \%)$ & $125(51.2 \%)$ & ${ }^{\dagger} p=0.004$ \\
\hline HVI por IMV $(n=160)(n-\%)$ & $97(60.6 \%)$ & $25(47.1 \%)$ & $72(67.3 \%)$ & ${ }^{\dagger} p=0.022$ \\
\hline Valvulpatías & $204(58.3 \%)$ & $57(53.7 \%)$ & $147(60.2 \%)$ & tNS \\
\hline Insuficiencia mitral (n-\%) & $120(34.3 \%)$ & $26(24.5 \%)$ & $94(38.5 \%)$ & ${ }^{\dagger} p=0.015$ \\
\hline Estenosis mitral (n-\%) & $3(0.85 \%)$ & $1(0.9 \%)$ & $2(0.8 \%)$ & tNS \\
\hline Insuficiencia aórtica (n-\%) & $83(23.7 \%)$ & $13(12.3 \%)$ & $70(28.6 \%)$ & ${ }^{\dagger} p=0.001$ \\
\hline Estenosis aórtica (n-\%) & $19(5.4 \%)$ & $5(4.7 \%)$ & $14(5.7 \%)$ & tNS \\
\hline Calcificaciones valvulares (n-\%) & $71(20.4 \%)$ & $10(9.5 \%)$ & $61(25.1 \%)$ & ${ }^{\dagger} p=0.001$ \\
\hline
\end{tabular}

Los valores de las variables continuas se expresan como mediana (percentil 25-75).

*Test Mann-Whitney.

${ }^{\dagger} \mathrm{T}$ Test $\mathrm{Chi}^{2}$

Al: aurícula izquierda; DDVI: diámetro diastólico del ventrículo izquierdo; FEVI: fracción de eyección del ventrículo izquierdo; IMV: índice de masa ventricular;

HVI: hipertrofia ventricular izquierda; PAD: presión arterial diastólica; PAS: presión arterial sistólica; PP: espesor de la pared posterior; SVI: espesor del septum interventricular izquierdo; RWT: espesor relativo de pared de ventrículo izquierdo. 
Tabla 3. Regresión logística binaria. Análisis uni y multivariado de riesgo de hipertrofia del ventrículo izquierdo según la masa ventricular izquierda y las valvulopatías

\begin{tabular}{|c|c|c|c|c|}
\hline & Análisis univariado & & Análisis multivariado & \\
\hline HVI por masa ventricular ${ }^{10}$ & OR (IC 95\%) & $\mathbf{p}$ & OR (IC 95\%) & $\mathbf{p}$ \\
\hline Sexo [ref. femenino] & $1.176(0.775-1.782)$ & 0.446 & - & - \\
\hline Edad (años) [cont] & $1.031(1.016-1.046)$ & 0.000 & $1.023(1.007-1.039)$ & 0.05 \\
\hline HTA [ref. No] & $1.667(1.071-2.595)$ & 0.024 & $1.446(0.914-2.287)$ & 0.115 \\
\hline Diabetes [ref. No] & $1.687(1.053-2.704)$ & 0.024 & $1.644(1.008-2.689)$ & 0.046 \\
\hline $\mathrm{Hb}(\mathrm{g} / \mathrm{dl})$ [cont] & $1.592(0.564-4.681)$ & 0.398 & - & - \\
\hline \multirow[t]{2}{*}{ ERC [ref. $\left.\mathrm{FGe} \geq 60 \mathrm{ml} / \mathrm{min} / 1.73 \mathrm{~m}^{2}\right]$} & $1.002(1.001-3.004)$ & 0.002 & $1.002(1.000-1.003)$ & 0.048 \\
\hline & Análisis univariado & & Análisis multivariado & \\
\hline Insuficiencia mitral & OR (IC 95\%) & $\mathbf{p}$ & OR (IC 95\%) & $\mathbf{p}$ \\
\hline Sexo [ref. femenino] & $1.345(0.862-2.099)$ & 0.192 & - & - \\
\hline Edad (años) [cont] & $1.04(1.026-1.062)$ & 0.000 & $1.041(1.023-1.060)$ & 0.000 \\
\hline HTA [ref. No] & $1.731(0.459-1.166)$ & 0.189 & - & - \\
\hline Diabetes [ref. No] & $0.827(0.503-1.359)$ & 0.454 & - & - \\
\hline \multirow[t]{2}{*}{ ERC [ref. FGe $\geq 60 \mathrm{ml} / \mathrm{min} / 1.73 \mathrm{~m}^{2}$ ] } & $1.002(1.000-1.003)$ & 0.014 & $1.001(0.999-1.002)$ & 0.308 \\
\hline & Análisis univariado & & Análisis multivariado & \\
\hline Insuficiencia aórtica & OR (IC 95\%) & $\mathbf{p}$ & OR (IC 95\%) & $\mathbf{p}$ \\
\hline Sexo [ref. femenino] & $0.586(0.356-0.965)$ & 0.036 & $0.770(0.449-1.323)$ & 0.344 \\
\hline Edad (años) [cont] & $1.080(1.054-1.107)$ & 0.000 & $1.074(1.047-1.102)$ & 0.000 \\
\hline HTA [ref. No] & $1.519(0.918-2.514)$ & 0.104 & - & - \\
\hline Diabetes [ref. No] & $0.874(0.502-1.521)$ & 0.634 & - & - \\
\hline ERC [ref. $\left.\mathrm{FGe} \geq 60 \mathrm{ml} / \mathrm{min} / 1.73 \mathrm{~m}^{2}\right]$ & $1.003(1.001-1.005)$ & 0.001 & $1.002(0.999-1.004)$ & 0.137 \\
\hline
\end{tabular}

ERC: enfermedad renal crónica; FGe: filtrado glomerular estimado por la fórmula CKD EPI; Hb: hemoglobinemia; HTA: hipertensión arterial; HVI: hipertrofia ventricular izquierda.

significativamente asociadas a ERCIII-V (test MannWhitney, $\mathrm{p}<0.05$ ) (Tabla 2).

La mediana (Pc 25-75) de masa del VI fue de $157 \mathrm{~g}$ (120-200) en ERC I-II y de $195 \mathrm{~g}$ (156-234) en ERC III-V (test Mann Whitney, $\mathrm{p}<0.05$ ) (Tabla 2). En el análisis multivariado de factores de riesgo para HVI (evaluada por masa del VI), la edad, diabetes y ERC estadio III-V fueron factores asociados independientemente (Tabla 3).

Se calculó el IMV en 160 casos (con datos de talla y peso), la mediana ( $P c$ 25-75) fue de $102 \mathrm{~g} / \mathrm{m}^{2}$ (86.5127.5), significativamente mayor en ERC III-V (Tabla 2). El RWT fue de 0.42 (0.37-0.48) en la población global, sin diferencia según el estadio de la ERC. En el análisis de la geometría ventricular izquierda (Fig. 1) (Tabla 4) se destaca que el FGe y la FEVI fueron significativamente menores en el grupo con HVI excéntrica y la presión arterial diastólica (PAD) más elevada en el grupo con HVI concéntrica.

El área de la Al fue significativamente mayor en el grupo ERC III-V, $21 \mathrm{~mm}^{2}$ (18-24) vs. $18 \mathrm{~mm}^{2}$ (15-22), al igual que el diámetro de la $\mathrm{Al}, 38 \mathrm{~mm}$ (34-42) vs. 35 mm (31-39) (test Mann-Whitney, p < 0.05) (Tabla 2).

El $58.3 \%$ de los pacientes presentó algún tipo de valvulopatía, en particular insuficiencia mitral (34.3\%) e insuficiencia aórtica (23.7\%). Ambas fueron significativamente más frecuentes en ERC estadio III-V $\left(\mathrm{Chi}^{2}, \mathrm{p}<0.05\right)$, al igual que las calcificaciones valvulares que se observaron en el $25.1 \%$ de los pacientes $\left(\mathrm{Chi}^{2}, \mathrm{p}<0.05\right)$. En el análisis multivariado, solo la edad 


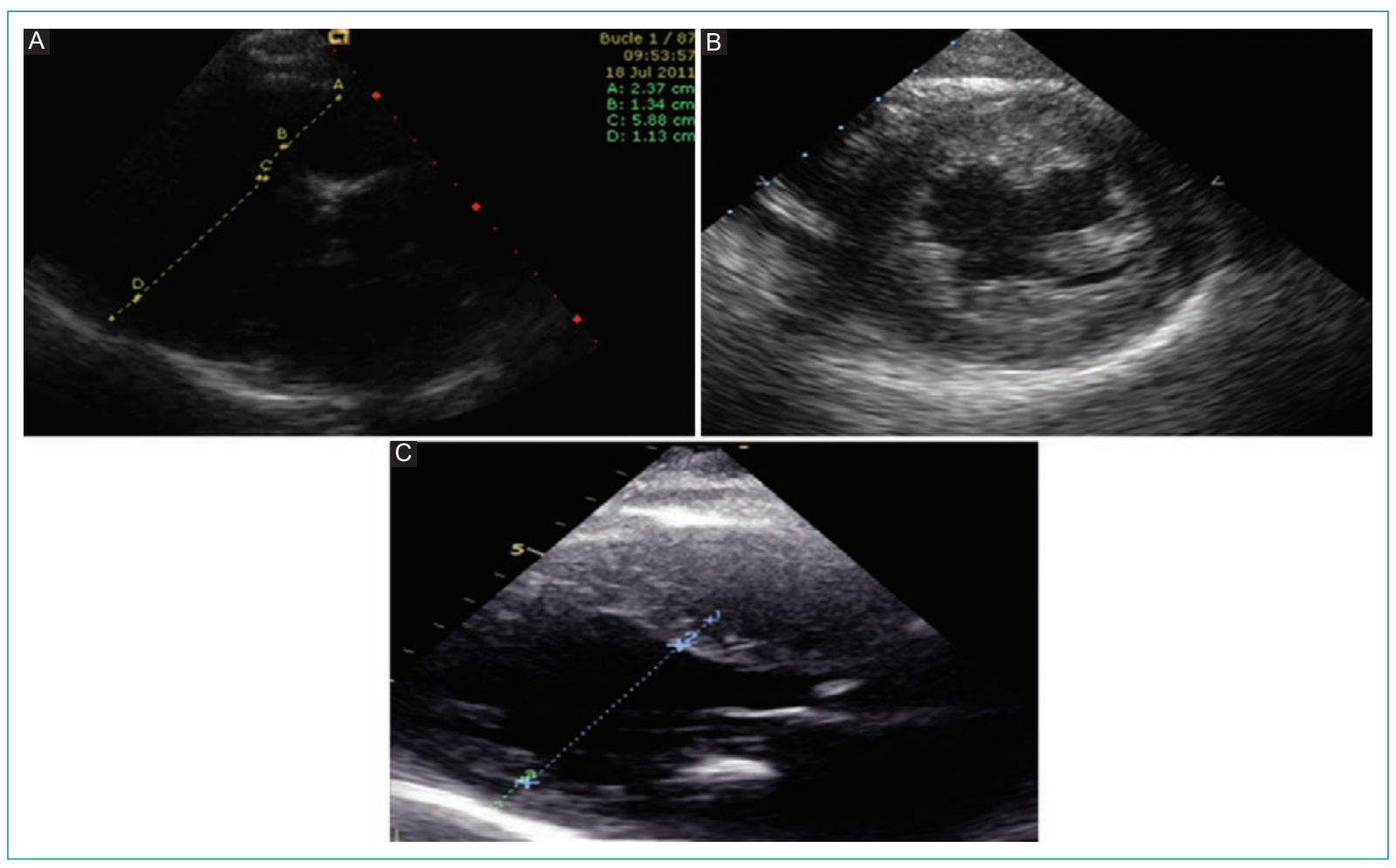

Figura 1. Geometría del ventrículo izquierdo (VI). Factores de riesgo. A: eje largo paraesternal; HVI excéntrica SIV, $13 \mathrm{~mm}$; PP, $11 \mathrm{~mm}$; masa, $139 \mathrm{~g} / \mathrm{m}^{2}$; RWT, 0.37. B: eje corto paraesternal; HVI concéntrica SIV, 14 mm; PP, 14 mm; masa, $143 \mathrm{~g} / \mathrm{m}^{2}$; RWT, 0.58. C: eje largo paraesternal; remodelado concéntrico SIV, 10 mm; PP, $10 \mathrm{~mm} ; \mathrm{masa}, 100 \mathrm{~g} / \mathrm{m}^{2}$; RWT, 0.38. Calcificación anillo mitral. PP: pared posterior; RWT: espesor relativo de pared de ventrículo izquierdo; SIV: septum iterventricular; VI: ventrículo izquierdo.

Tabla 4. Geometría del ventrículo izquierdo $(n=160)$

\begin{tabular}{|l|c|c|c|c|c|}
\hline & Normal & Remodelado concéntrico & HVI concéntrica & HVI excéntrica & * p \\
\hline N (5\%) & $35(21.8)$ & $28(17.5)$ & $45(28,1)$ & $52(32.5)$ \\
\hline Edad & $64(54-72)$ & $58.5(45-79)$ & $66(58-76)$ & $66.5(57-75)$ & NS \\
\hline PAS & $130(110-148)$ & $125(115-149)$ & $141(119-159)$ & $131(119-149)$ & NS \\
\hline PAD & $70(60-80)$ & $74(60-82)$ & $80(70-90)$ & $71(65-82)$ & 0.049 \\
\hline FGe & $58.8(44.6-74.2)$ & $60.6(32.6-74.8)$ & $49.1(36.1-58.4)$ & $44.6(31.1-60.5)$ & 0.039 \\
\hline FEVI & $60(55-65)$ & $60(60-65)$ & $60(55-65)$ & $57-5(43-60)$ & 0.004 \\
\hline
\end{tabular}

Mediana (pc 25-75) de variables simultáneas al ecocardiograma Doppler. *Test de Kruskall-Wallis. Significativo p < 0.05. FEVI: fracción de eyección del ventrículo izquierdo; FGe: filtrado glomerular estimado; HVI: hipertrofia ventricular izquierda; PAD: presión arterial diastólica; PAS: presión arterial sistólica.

fue un factor de riesgo significativo independiente de valvulopatía (Tablas 2 y 3).

\section{Discusión}

Es bien conocido que las alteraciones cardiovasculares en pacientes con ERC son muy frecuentes, y representan la principal causa de muerte ${ }^{1-3,8,10}$, por lo que el análisis de los factores de riesgo es relevante. En el presente estudio analizamos los datos ecocardiográficos y bioquímicos de un grupo de 350 pacientes con diagnóstico de nefropatía y en seguimiento por un equipo nefrológico multidisciplinario². Observamos que la frecuencia de las alteraciones cardíacas 
(auriculares, ventriculares, valvulares) era mayor en la ERC en estadios III-V, y que el menor FGe fue un factor de riesgo independiente de HVI, lo cual condice con la literatura existente $e^{4-10,14-17}$.

En un estudio previo, nacional, del PSRU ${ }^{8}$ se observó una tasa de nuevo evento cardiovascular de 10.1 por 100 pacientes-año y una tasa de mortalidad global de 6.2 (2.03 por 100 paciente-año de causa cardiovascular), con una asociación de evento cardiovascular y mortalidad con factores de riesgo tradicionales, así como vinculados a la ERC.

El rango de edad es muy amplio, con una mediana de 65 años, la mayoría (69.7\%) con ERC estadio III-V. La proteinuria (reconocido factor de progresión de ERC y de riesgo cardiovascular) fue inferior a $0.5 \mathrm{~g} / \mathrm{l}$ en la mayoría y el control de la presión arterial fue aceptable (medianas de presión arterial sistólica (PAS)/PAD 130/76 mmHg, sin diferencia según el estadio de ERC), así mismo, los niveles de hemoglobinemia, calcemia y fosforemia estaban en rangos objetivo (Tabla 2). Consideramos que el análisis retrospectivo de esta población, con aceptable control, puede presentar un sesgo de selección.

\section{Función ventricular}

La Guía Europea de Insuficiencia Cardíaca ${ }^{20}$ plantea una prevalencia del 1 a $2 \%$ en la población adulta, que aumenta a un $10 \%$ en los mayores de 70 años. La HTA y las valvulopatías son causas importantes de insuficiencia cardíaca, entre otras, y la ERC configura un factor de riesgo independiente ${ }^{4-6-10,21}$. En el presente estudio, la FEVI mediana fue del $60 \%$, pero el $10 \%$ de los pacientes tenía valores inferiores al $40 \%$ (5.7\% en ERC I-II y $11.9 \%$ en ERC III-V) (Tabla 2).

\section{Hipertrofia ventricular izquierda y dilatación auricular}

La HVI es un predictor independiente de mortalidad cardiovascular en pacientes con ERC identificado hace varias décadas ${ }^{4-10,14-17}$. En una cohorte japonesa de pacientes con $\mathrm{ERC}^{21}$, Nitta, et al. reportan como factores de riesgo independientes de HVI, el presentar antecedentes de ECV, la PAS, el índice de masa corporal y los niveles de calcemia. Los estudios CRIC ${ }^{4,5}$ y CAS$\mathrm{CADE}^{22}$ han demostrado la elevada frecuencia de HVI y los cambios evolutivos en la ERC. Paoletti ${ }^{10}$, et al. en una población con ERC observaron que la HVI era un fuerte predictor de eventos cardiovasculares y de menor sobrevida renal, y se asociaba con HTA nocturna.
Se ha demostrado una prevalencia de hasta un $75 \%$ en pacientes que inician terapia de reemplazo renal, principalmente en los añosos ${ }^{23}$. En el presente estudio la frecuencia de HVI fue elevada (Tabla 2), principalmente cuando se definía por masa del VI e IMV, lo que evidencia la importancia de estos cálculos para un diagnóstico oportuno. La HVI se asoció a mayor edad, y menor FGe, independientemente de la HTA y la diabetes (Tabla 3), lo que destaca el papel de la ERC como factor de riesgo relevante.

El análisis de la geometría del $\mathrm{V}^{11}$ en un subgrupo (Fig. 1) (Tabla 4) evidencia que solo el $21.8 \%$ presentaban un ventrículo normal, el $28.1 \%$ hipertrofia concéntrica y el $32.5 \%$ hipertrofia excéntrica. La hipertrofia excéntrica se caracteriza por un incremento menor del espesor de la pared ventricular y un aumento del volumen de la cavidad, probablemente causado por sobrecarga de volumen. Se ha planteado que el tamaño de los miocitos aumenta predominantemente en largo debido al agregado de sarcómeros en serie, lo que origina este tipo de remodelado cardíaco $^{10}$. En la población estudiada, la disminución del FGe y/o la alta prevalencia de valvulopatías regurgitantes podrían explicarlo ${ }^{21}$ (Tablas 2 y 4) (Fig. 1). El subgrupo con HVI excéntrica tenía una FEVI y un FGe significativamente menores, en tanto que aquellos con HVI concéntrica tenían una PAD significativamente mayor. En un análisis fisiopatológico, el aumento de la poscarga (mayor PAD) contribuiría a la HVI concéntrica, en tanto que los efectos de la uremia (toxinas, sobrecarga de volumen) contribuirían a la HVI excéntrica asociada a una disminución de la FEVI. Estos datos destacan la importancia del cálculo de la masa ventricular, ya que permitiría detectar un subgrupo con alteraciones cardíacas que quedaría subdiagnosticado si solo se observara el espesor parietal, por ejemplo, y enfatiza el papel de los factores vinculados a la uremia per se en las anomalías miocárdicas ${ }^{6,7,10,22}$. También se observaron anomalías auriculares, tanto el área como el diámetro de la aurícula izquierda, que fueron mayores en los estadios ERC III-V (Tabla 2).

\section{Valvulopatías}

Finalmente se destacan las anomalías valvulares detectadas, menos reportadas en la literatura ${ }^{17,21-25}$, principalmente insuficiencia mitral y aórtica, ambas significativamente más frecuentes en la ERC III-V (Tablas 2 y 3), aunque en el análisis multivariado de riesgo, el único factor independiente fue la mayor edad (Tabla 3). Existen pocos reportes de 
prevalencia de valvulopatías en ERC y las Guías terapéuticas ${ }^{20}$ no hacen recomendaciones específicas. Samad, et al. ${ }^{17}$ recientemente analizaron un vasto registro ecocardiográfico (realizados a demanda de la situación clínica), que incluye pacientes con ERC, y concluye que las valvulopatías del VI son altamente prevalentes en la ERC, y se asociaron con mayor mortalidad. No encontramos reportes de registros ecocardiográficos de valvulopatías de control como el presente y tampoco se ha descrito si la detección precoz de estas anomalías determinara cambios de conducta preventivos.

Las calcificaciones valvulares y vasculares, son muy frecuentes en los pacientes portadores de ERC, incluso previo a su ingreso a terapia de reemplazo renal, y tienden a progresar después de su ingreso a diálisis. Sayarlioglu ${ }^{23}$, et al. reportan que el $43 \%$ de los pacientes con ERC en hemodiálisis tienen calcificaciones valvulares (23\% mitral y $21.7 \%$ aórtica). Estas alteraciones se describen como factor de riesgo independiente de muerte de cualquier causa y mortalidad cardiovascular ${ }^{23,24}$. Los reportes en ERC previo a diálisis, como el presente estudio, son escasos. Las calcificaciones valvulares fueron frecuentes $(20.4 \%)$, y significativamente más frecuentes en ERC estadio III-V (Tabla 2). Múltiples estudios demuestran que las alteraciones del metabolismo óseo-mineral, y otros factores de la ERC, se relacionan con las calcificaciones extraóseas ${ }^{4-9,20-24}$, pero su análisis excede los objetivos del presente estudio.

Existen limitaciones en el análisis realizado por ser retrospectivo, lo cual determina que algunas variables tengan datos incompletos (talla), y el posible sesgo de selección ya mencionado, pero consideramos que aporta datos de interés de una población con elevada morbimortalidad cardiovascular.

\section{Conclusiones}

El análisis de ecocardiogramas de control en pacientes con ERC confirma la frecuencia de alteraciones morfológicas cardíacas, mayor en etapas avanzadas de la ERC, como disfunción ventricular izquierda, remodelación cardíaca (auricular y ventricular) y afectación valvular, esta última menos reconocida, al tiempo que destaca la importancia del cálculo del índice de masa ventricular.

Estos hallazgos pueden orientar el diseño de estrategias para el diagnóstico oportuno y cambios terapéuticos, que contribuyan a mejorar el pronóstico vital de esta población.

\section{Agradecimientos}

Los autores agradecen a todos los integrantes del equipo de Salud Renal del Hospital de Clínicas.

\section{Conflicto de intereses}

Los autores declaran no tener ningún conflicto de interés. La presente investigación no ha recibido ayudas específicas provenientes de agencias del sector público, sector comercial o entidades sin ánimo de lucro.

\section{Financiamiento}

El proyecto de investigación no recibió financiación específica. Los autores lo realizaron en el horario laboral financiado para investigación en sus cargos en la Facultad de Medicina, Universidad de la República, Uruguay.

\section{Responsabilidades éticas}

Protección de personas y animales. Los autores declaran que para esta investigación no se han realizado experimentos en seres humanos ni en animales.

Confidencialidad de los datos. Los autores declaran que han seguido los protocolos de su centro de trabajo sobre la publicación de datos de pacientes.

Derecho a la privacidad y consentimiento informado. Los autores han obtenido el consentimiento informado de los pacientes y/o sujetos referidos en el artículo. Este documento obra en poder del autor de correspondencia.

\section{Bibliografía}

1. U.S Renal Data System USDRS 2013 Annual Data Report: Atlas of Chronic Kidney disease and End-Stage Renal Disease in the United States. Bethesda, MD: National Institutes of Health, National Institute of Diabetes and Digestive and Kidney Diseases; 2013.

2. Schwedt E, Solá L, Ríos PG, Mazzuchi N; National Renal Healthcare Program. Improving the management of chronic kidney disease in Uruguay: a National Renal Healthcare Program. Nephron Clin Pract. 2010; 114(1):c47-59.

3. Tumlin JA, Costanzo MR, Chawla LS, Herzog CA, Kellum JA, Mccullough PA, Ronco C. Cardiorenal syndrome type 4: insights on clinical presentation and pathophysiology from the eleventh consensus conference of the Acute Dialysis Quality Initiative (ADQI). Contrib Nephrol. 2013; 182:158-73

4. Ricardo AC, Lash JP, Fischer MJ, Lara C, Budoff M, Keane M, et al. Cardiovascular Disease Among Hispanics and Non-Hispanics in the Chronic Renal Insufficiency Cohort (CRIC) Study. Clin J Am Soc Neph. 2011;6: 2121-31.

5. Park M, Hsu CY, Li Y, Mishra RK, Keane M, Rosas SE, et al. Chronic Renal Insufficiency Cohort (CRIC) Study Group: Association between kidney function and subclinical cardiac abnormalities in CKD. J Am Soc Nephrol. 2012;23:1725-34.

6. Herzog C, Asinger R, Berger A, Charytan D, Diez J, Hart R, et al. Cardiovascular disease in chronic kidney disease. A clinical update from Kidney Disease: Improving Global Outcomes (KDIGO). Kidney Int. 2011;80:572-86 
7. Muntner $P$, Judd $S$, Gao L, Gutierrez $O$, Rizk D, Mcclellan $W$ et al. Cardiovascular risk factors in CKD associate with both ESRD and mortality. J Am Soc Nephrol. 2013;24:1159-65.

8. Subiza A, Odriozola M, Ríos P, Lamadrid V, Mazzuchi N, Gadola L. Riesgo cardiovascular en la enfermedad renal crónica. Rev Urug Cardiol. 2016;31:206-18

9. Ha SK, Park HS, Kim SJ, Park CH, Kim DS, Kim HS. Prevalence and Patterns of Left Ventricular Hypertrophy in Patients with Predialysis Chronic Renal Failure. J Korean Med Sci. 1998;13:488-94

10. Paoletti E, De Nicola LM, Gabbai F, Chiodini P, Ravera M, Pieracci L, et al. Associations of Left Ventricular Hypertrophy and Geometry with adverse outcomes in patients with CKD and hypertension. Clin J Am Soc Nephrol. 2016;11:271-9.

11. Lang RM, Badano LP, Mor-Avi V, Afilalo J, Armstrong A, Ernande L, et al. Recommendations for Cardiac Chamber Quantification by Echocardiography in Adults: An Update from the American Society of Echocardiography and the European Association of Cardiovascular Imaging. J Am Soc Echocardiogr. 2015;28:1-39.

12. Weiner DE, Tighiouart H, Elsayed EF, Griffith JL, Salem DM, Levey AS et al. Inflammation and cardiovascular events in individuals with and without chronic kidney disease. Kidney Int. 2008;73:1406-12.

13. Briet M, Schiffrin EL. Aldosterone: effects on the kidney and cardiovascular system. Nat Rev Nephrol. 2010;6:261-73.

14. Bovera J, Górriz JL, Ureña-Torresc P, Lloreta MJ, Ruiz-García C Dasilvaa I, et al. Detección de las calcificaciones cardiovasculares: ¿una herramienta útil para el nefrólogo? Nefrologia (Madr). 2016;36: 587-96.

15. Sánchez-Peralesa C, Vázquez Ruiz De Castroviejo E, García-Cortés MJ, Biechya M, Gil-Cunquero JM, Borrego-Hinojosa J, et al. Las calcificaciones valvulares al inicio de diálisis predicen la aparición de eventos cardiovasculares en la evolución. Nefrologia (Madr). 2015;35:157-63.

16. Liu M, Li XC, Lu L, Cao Y, Sun RR, Chen S, et al. Cardiovascular disease and its relationship with chronic kidney disease. Eur Rev Med Pharmacol Sci. 2014;18:2918-26.
17. Samad Z, Sivak J, Phelan M, Schulte P, Patel U, Velazquez E. Prevalence and Outcomes of left-sided valvular heart disease associated with chronic kidney disease. J Am Heart Assoc. 2017: 6:e006044.

18. Levey A, Eckardt K, Tsukamoto Y, Levin A, Coresh J, Rossert J, et al. Definition and classification of chronic kidney disease: A position statement from Kidney Disease: Improving Global Outcomes (KDIGO). Kidney Int. 2005;67:2089-100.

19. Lenfant C, Chobanian AV, Jones DW, Roccella EJ; Joint National Committee on the Prevention, Detection, Evaluation, and Treatment of High Blood Pressure. Seventh report of the Joint National Committee on the Prevention, Detection, Evaluation, and Treatment of High Blood Pressure (JNC 7): resetting the hypertension sails. Hypertension. 2003;41:1178-9.

20. Ponikowski P, Voors AA, Anker SD, Bueno H, Cleland JGF, Coats AJS, Falk V. ESC Guidelines for the diagnosis and treatment of acute and chronic heart failure. 2016 ESC Guidelines for the diagnosis and treatment of acute and chronic heart failure: The Task Force for the diagnosis and treatment of acute and chronic heart failure of the European Society of Cardiology (ESC) Developed with the special contribution of the Heart Failure Association (HFA) of the ESC. Eur Heart J. 2016;37(27):2129-200.

21. Nitta K, limuro S, Imai E, Matsuo S, Makino H, Akizawa T, et al. Risk factors for increased left ventricular hypertrophy in patients with chronic kidney disease. Clin Exp Nephrol. 2013:17:730-42.

22. Cai QZ, Lu XZ, Lu Y, Wang AY. Longitudinal changes of cardiac structure and function in CKD (CASCADE study). J Am Soc Nephrol. 2014; 25:1599-1608.

23. Sayarlioglu H, Acar G, Sahin M, Altunoren O, Coskun Yavuz Y, Nacar AB, et al. Prevalence and risk factors of valvular calcification in hemodialysis patients. Iran J Kidney Dis. 2013;7(2):129-34.

24. Leibowitz D. Left ventricular hypertrophy and chronic renal insufficiency in the elderly. Cardio Renal Med. 2014;4:168-75.

25. Panuccio V, Tripepi R, Tripepi G, Mallamaci F, Benedetto FA, Cataliotti A Bellanuova I, Giacone G, Malatino LS, Zoccali C. Heart valve calcifications, survival, and cardiovascular risk in hemodialysis patients. Am J Kidney Dis. 2004;43:479-84. 